\title{
Noise-induced switching near a depth two heteroclinic network and an application to Boussinesq convection
}

\author{
Peter Ashwin ${ }^{1}$ and Olga Podvigina ${ }^{2, a)}$ \\ ${ }^{1}$ Mathematics Research Institute, University of Exeter, Exeter EX4 4QF, United Kingdom \\ ${ }^{2}$ International Institute of Earthquake Prediction Theory and Mathematical Geophysics, \\ 84/32 Profsoyuznaya St., 117997 Moscow, Russian Federation
}

(Received 4 September 2009; accepted 10 May 2010; published online 30 June 2010)

\begin{abstract}
We investigate the robust heteroclinic dynamics arising in a system of ordinary differential equations in $\mathbb{R}^{4}$ with symmetry $D_{4} \ltimes\left(Z_{2}\right)^{2}$. This system arises from the normal form reduction of a $1: \sqrt{2}$ mode interaction for Boussinesq convection. We investigate the structure of a particular robust heteroclinic attractor with "depth two connections" from equilibria to subcycles as well as connections between equilibria. The "subcycle" is not asymptotically stable, due to nearby trajectories undertaking an "excursion," but it is a Milnor attractor, meaning that a positive measure set of nearby initial conditions converges to the subcycle. We investigate the dynamics in the presence of noise and find a number of interesting properties. We confirm that typical trajectories wind around the subcycle with very occasional excursions near a depth two connection. The frequency of excursions depends on noise intensity in a subtle manner; in particular, for anisotropic noise, the depth two connection may be visited much more often than for isotropic noise, and more generally the long term statistics of the system depends not only on the noise strength but also on the anisotropy of the noise. Similar properties are confirmed in simulations of Boussinesq convection for parameters giving an attractor with depth two connections. () 2010 American Institute of Physics. [doi:10.1063/1.3439320]
\end{abstract}

It is well known that symmetries can cause rather strange and uncharacteristic behavior in smooth dynamical systems. A particularly counterintuitive phenomenon is the appearance of robust heteroclinic attractors, ${ }^{14}$ where trajectories of symmetric systems visit a sequence of saddle points, such that the sequence of residence times in a given neighborhood of a saddle tends to infinity. In the presence of noise and/or symmetry breaking perturbations the sequence of residence times attains a finite mean value after a transient. ${ }^{25}$ This paper explores a particular example of a heteroclinic attractor in $\mathbb{R}^{4}$ with a depth two connection; ${ }^{3}$ this means that some connections in the attractor are not between equilibria, but between an equilibrium and a heteroclinic subcycle. For the example, we find that the "subcycle" is an attractor in its own right; it attracts most, but not all, nearby trajectories. Some trajectories starting arbitrarily close to the subcycle undergo an "excursion" before returning. The approach to the attractor can therefore show periods of approach toward to subcycle interrupted by the excursions. The frequency of the excursions per cycle is found to depend on the details of the added noise.

\section{INTRODUCTION}

Robust heteroclinic cycles can be attractors in the sense of Milnor, while failing to be asymptotically stable. They may attract trajectories starting at a positive measure set

\footnotetext{
a) Also at CNRS, Laboratoire Cassiopée, Observatoire de la Côte d'Azur, BP 4229, 06304 Nice Cedex 4, France.
}

nearby in phase space while repelling another, complementary, set-this phenomenon has been called essential asymptotic stability. ${ }^{15,17}$ In addition, robust heteroclinic cycles may share a common connection to form a network with competition between different cycles. In Ref. 13 such a system is studied, where both cycles can be simultaneously Milnor attractors. One may find heteroclinic networks where no subcycle is an attractor, but the whole network is attracting and asymptotic behavior involves chaotic switching near the network. ${ }^{1,10,11,21,22}$

Numerical and analytical studies in Ref. 20 found an open region in parameter space for an eight-dimensional (8D) symmetric system of ordinary differential equations (ODEs), where the only attractor is a robust depth two heteroclinic network involving a variety of saddle equilibria classified according to their symmetries. In this paper we consider a considerable simplification of the $8 \mathrm{D}$ system of Ref. 20 to a four-dimensional (4D) subspace, such that the simplified system retains the essential dynamical properties of the depth two attractor.

The addition of noise to such attractors can have a dramatic effect: typical $\omega$-limit sets of trajectories become much larger and a simple argument says that typical trajectories approaching a heteroclinic network will recurrently explore all unstable manifolds of points on the network. The dependence of trajectory properties on noise amplitude is of interest and has been explored in Refs. 2, 21, and 24, but so far not for any networks with depth two connections.

In this paper we make three observations concerning the dynamics with noise. First, the addition of noise to a system 


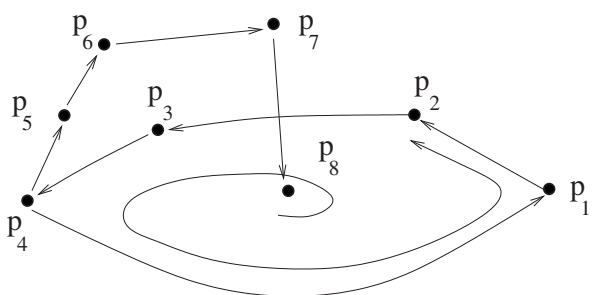

FIG. 1. Chawanya's example of a network with a "child cycle"-a heteroclinic network that is not of depth one. Observe that (a) the unstable manifold of the equilibrium $p_{8}$ is a connection that accumulates on a simple heteroclinic cycle connecting $\left\{p_{1}, p_{2}, p_{3}, p_{4}\right\}$, and (b) the unstable manifolds of $p_{4}$ and $p_{8}$ are two-dimensional.

with a stable depth two attractor may appear to stabilize the subcycle. This is similar to observations of Ref. 2 that switching between competing cycles within a heteroclinic network may become more, rather than less, regular on addition of noise. Second, anisotropic noise may have a significant influence on how often excursions are made from the subcycle. Third, we verify that this behavior appears in simulations of problem in Boussinesq convection.

The paper is organized as follows: we give some definitions in Sec. I A and introduce in Sec. I B the particular set of four coupled ODEs that we study, briefly motivating where they arise in a normal form problem. We discuss some dynamics and attractors of this system. Section II studies the dynamics of this ODE for specific parameter values that give a robust heteroclinic network with depth two.

Section III considers (both numerically and analytically) the influence of noise on this cycle and investigates the frequency of excursions from the subcycle as a function of the noise amplitude in different components of the system. Section IV exhibits numerical evidence of the presence of these cycles in a problem from Boussinesq convection. Finally, we discuss implications for more general systems in Sec. V as well as some open questions concerning depth two networks for these and related systems.

\section{A. Heteroclinic networks and depth}

Suppose we have an ODE $\dot{x}=f(x)$ for $x \in \mathbb{R}^{n}$ with $f$ smooth that generates a smooth flow $\Phi_{T}(x)$. We say a compact invariant chain recurrent $\operatorname{set}^{23} \Sigma$ is a heteroclinic network of depth one (between equilibria) if the following hold:

- $\Sigma$ contains a finite set $\left\{x_{i}\right\}$ of hyperbolic equilibria.

- If $y \in \Sigma \backslash\left\{x_{i}\right\}$ then $y \in W^{u}\left(x_{i_{1}}\right) \cap W^{s}\left(x_{i_{2}}\right)$ for some $i_{1}$, $i_{2}$, i.e., $\lim _{T \rightarrow-\infty} \phi_{T}(y)=x_{i_{1}}$ and $\lim _{T \rightarrow \infty} \phi_{T}(y)=x_{i_{2}}$.

We say a heteroclinic network is a simple heteroclinic cycle if it is topologically a circle. This means that all equilibria have one-dimensional unstable manifolds within $\Sigma$, and $\Sigma$ can, with a suitable reordering, be seen as a union of $n$ equilibria and $n$ connections from one equilibrium to the next. As a result, a simple heteroclinic cycle has depth one. However, this notion of heteroclinic network does not include all possible situations that can appear even if we restrict to robust cases. Figure 1 schematically shows an example of Chawanya $^{7}$ from a game dynamical system-note the presence of a connection that is not from equilibrium to equilib- rium, but rather from equilibrium to subcycle.

As a way to understand the dynamics of more general heteroclinic networks, the concept of depth of a heteroclinic network was introduced by Ref. 3. We say $\Sigma$ is a heteroclinic network of depth two if it is a compact invariant chain recurrent set such that the following hold:

- $\Sigma$ contains a finite set $\left\{x_{i}\right\}$ of hyperbolic equilibria.

- $\Sigma^{\prime}=\cup_{x \in \Sigma} \overline{\alpha(x) \cup \omega(x)}$ is a union of heteroclinic networks of depth one and equilibria.

We assume that $\Sigma \neq \Sigma^{\prime} \neq\left\{x_{i}\right\}$.

Almost all previously documented heteroclinic networks are of depth one. Nonetheless, in symmetric systems of dimension higher than three, previous work $^{3}$ suggests that depth two networks can be robust, and that paper gives an example in nine dimensions; the example of Ref. 7 is in five dimensions, meaning that the example in this paper (in four dimensions) is of lower dimension than either. Finally, we mention that Ref. 8 gives an example flow on a manifold with boundary in three dimensions that has a robust depth two heteroclinic network; however, the latter is presumably not robust for any compact symmetry group action.

\section{B. A model with a depth two heteroclinic network}

We consider a system that is a restriction to an $\mathbb{R}^{4}$ subspace of a $\mathrm{C}^{4}$ normal form for a mode interaction present in a Boussinesq convection problem. ${ }^{26}$ Consider the dynamics of the following system of four coupled ODEs on $\left(x_{1}, x_{2}, y_{1}, y_{2}\right) \in \mathbb{R}^{4}$ with added noise:

$$
\begin{aligned}
& \dot{x}_{1}=\left(\lambda_{1}+B_{1} x_{1}^{2}+B_{2} x_{2}^{2}+B_{3} y_{1}^{2}+B_{4} y_{2}^{2}\right) x_{1}+\eta_{1}, \\
& \dot{x}_{2}=\left(\lambda_{1}+B_{1} x_{2}^{2}+B_{2} x_{1}^{2}+B_{3} y_{2}^{2}+B_{4} y_{1}^{2}\right) x_{2}+\eta_{2}, \\
& \dot{y}_{1}=\left(\lambda_{2}+B_{5} y_{1}^{2}+B_{6} y_{2}^{2}+B_{7} x_{1}^{2}+B_{8} x_{2}^{2}\right) y_{1}+\eta_{3}, \\
& \dot{y}_{2}=\left(\lambda_{2}+B_{5} y_{2}^{2}+B_{6} y_{1}^{2}+B_{7} x_{2}^{2}+B_{8} x_{1}^{2}\right) y_{2}+\eta_{4} .
\end{aligned}
$$

The $\eta_{i}$ represent independent additive white noise processes. The real quantities $\lambda_{i}$ and $B_{i}$ are system parameters and we assume the noise is independent, $\left\langle\eta_{i} \eta_{j}\right\rangle=0$ for $i \neq j$, and has amplitude $\left\langle\eta_{i}(t) \eta_{i}\left(t^{\prime}\right)\right\rangle=\left(\epsilon_{i}\right)^{2} \delta\left(t-t^{\prime}\right)$. We say the noise is isotropic if $\epsilon_{i}=\epsilon$ independent of $i$, otherwise it is anisotropic. The system (1) is symmetric (equivariant) under the action of the transformations

$$
\begin{aligned}
& \rho\left(x_{1}, x_{2}, y_{1}, y_{2}\right)=\left(x_{2}, x_{1}, y_{2}, y_{1}\right), \\
& \kappa\left(x_{1}, x_{2}, y_{1}, y_{2}\right)=\left(-x_{1}, x_{2},-y_{1}, y_{2}\right), \\
& \kappa_{1}\left(x_{1}, x_{2}, y_{1}, y_{2}\right)=\left(x_{1}, x_{2},-y_{1}, y_{2}\right), \\
& \kappa_{2}\left(x_{1}, x_{2}, y_{1}, y_{2}\right)=\left(x_{1}, x_{2}, y_{1},-y_{2}\right)
\end{aligned}
$$

that generate the group $\mathrm{D}_{4} \ltimes\left(\mathbb{Z}_{2}\right)^{2}$, where $\mathbb{D}_{4}=\langle\{\rho, \kappa\}\rangle$ and $\left(\mathbb{Z}_{2}\right)^{2}=\left\langle\left\{\kappa_{1}, \kappa_{2}\right\}\right\rangle$ (note that this is not a minimal set of generators as $\left.\kappa_{2}=\rho^{-1} \kappa_{1} \rho\right)$.

We will concentrate on a depth two network that appears robustly in the dynamics of the system (1). First, we list in Table I the various equilibria that exist in invariant subspaces 
TABLE I. Invariant subspaces and nontrivial equilibria involved in the heteroclinic attractor for Eq. (1), shown with eigenvectors and eigenvalues determining their stability. The same labels as in Ref. 20 are employed except that AR corresponds to AR1 and WR to WR2 in that reference. Typical points are given in terms of the coordinates $\left(x_{1}, x_{2}, y_{1}, y_{2}\right)$. The last column gives the eigenvalues (or just their signs) for the particular parameter values given in Table II.

\begin{tabular}{|c|c|c|c|c|}
\hline Subspace & $\left(x_{1}, x_{2}, y_{1}, y_{2}\right)$ & Eigenvectors & $\begin{array}{l}\text { Eigenvalues, } \\
\text { formulae }\end{array}$ & $\begin{array}{c}\text { Eigenvalues, } \\
\text { particular values }\end{array}$ \\
\hline LR & $\xi_{\mathrm{LR}}=(x, 0,0,0)$ & $(q, 0,0,0)$ & $-2 \lambda_{1}$ & $-r_{1} \approx-0.45751$ \\
\hline \multirow[t]{3}{*}{ (Large rolls) } & $x^{2}=-\lambda_{1} / B_{1}$ & $(0, q, 0,0)$ & $\lambda_{1}\left(B_{1}-B_{2}\right) / B_{1}$ & $-c_{12} \approx-0.10513$ \\
\hline & & $(0,0,0, q)$ & $\lambda_{2}+B_{8} x^{2}$ & $e_{14} \approx 0.11721$ \\
\hline & & $(0,0, q, 0)$ & $\lambda_{2}+B_{7} x^{2}$ & $-c_{13} \approx-0.78153$ \\
\hline SS & $\xi_{\mathrm{SS}}=(0,0, x, 0)$ & $(0,0, q, 0)$ & $-2 \lambda_{2}$ & $-r_{4} \approx-1.94697$ \\
\hline \multirow[t]{3}{*}{ (Small squares) } & $x^{2}=-\lambda_{1} / B_{5}$ & $(0,0,0, q)$ & $\lambda_{2}\left(B_{5}-B_{6}\right) / B_{5}$ & $e_{34} \approx 0.55628$ \\
\hline & & $(q, 0,0,0)$ & $\lambda_{1}+B_{3} x^{2}$ & $e_{24} \approx 1.66908$ \\
\hline & & $(0, q, 0,0)$ & $\lambda_{1}+B_{4} x^{2}$ & $-c_{41} \approx-1.38664$ \\
\hline LS & $\xi_{\mathrm{LS}}=(x, x, 0,0)$ & $(q, q, 0,0)$ & $-2 \lambda_{1}$ & - \\
\hline \multirow[t]{2}{*}{ (Large squares) } & $x^{2}=-\lambda_{2} /\left(B_{1}+B_{2}\right)$ & $(q,-q, 0,0)$ & $2 \lambda_{1}\left(B_{2}-B_{1}\right) /\left(B_{2}+B_{1}\right)$ & + \\
\hline & & $\left(0,0, q_{1}, q_{2}\right)$ & $\lambda_{2}+\left(B_{7}+B_{8}\right) x^{2}$ &,+- \\
\hline SR & $\xi_{\mathrm{SR}}=(0,0, x, x)$ & $(0,0, q, q)$ & $-2 \lambda_{2}$ & - \\
\hline \multirow[t]{2}{*}{ (Small rolls) } & $x^{2}=-\lambda_{2} /\left(B_{5}+B_{6}\right)$ & $(0,0, q,-q)$ & $2 \lambda_{2}\left(B_{6}-B_{5}\right) /\left(B_{6}+B_{5}\right)$ & - \\
\hline & & $\left(q_{1}, q_{2}, 0,0\right)$ & $\lambda_{1}+\left(B_{3}+B_{4}\right) x^{2}$ &,++ \\
\hline $\mathrm{AR}$ & $\xi_{\mathrm{AR}}=\left(x_{1}, 0, x_{3}, x_{4}\right)$ & $\left(q_{1}, 0, q_{3}, q_{4}\right)$ & &,,+-- \\
\hline (Asymmetric rolls) & & $(0, q, 0,0)$ & & - \\
\hline WR & $\xi_{\mathrm{WR}}=\left(x_{1}, x_{1}, x_{3}, x_{3}\right)$ & $\left(q_{1}, q_{1}, q_{3}, q_{3}\right)$ & &,-- \\
\hline (Wavy rolls) & & $\left(q_{1},-q_{1}, q_{4},-q_{4}\right)$ & &,$++($ complex $)$ \\
\hline
\end{tabular}

forced by symmetries of the system; in the last two columns of the table we present eigenvalues determining the stability of the equilibria for a general set of parameter values and for the particular set given in Table II. We use the notation of Ref. 20 for convenience (LR refers to large rolls, SS to small squares, etc.). A list of connections between these equilibria for these parameters is presented in Table III.

Figure 2 shows some examples of attracting dynamics at parameter values in Table II in the presence of two different levels of noise in the $x_{i}$ and $y_{i}$ components. Our aim in Secs. II-IV is to describe and explain these dynamics in terms of a depth two heteroclinic cycle. The trajectories are shown projected onto various axes, with transients removed. We find that the subset of the phase space explored is clearly much larger in the second case. It depends strongly on the noise amplitude of the last two components, but weakly on the noise amplitude in the first two components. We will show that the structure of the heteroclinic attractor that organizes the dynamics is shown in Fig. 3, and typical time series are shown in Fig. 4. Note that larger noise in the last two components induces repeated "excursions" from a regular LR/SS cycle.

TABLE II. Parameter values that give the depth two heteroclinic attractor for Eq. (1); we note that $\lambda_{1}=\cos \theta, \lambda_{2}=\sin \theta$ where $\theta=1.34$ is a parameter used in Ref. 20 to unfold a mode interaction.

\begin{tabular}{lrlr}
\hline \hline$\lambda_{1}$ & 0.228752 & $\lambda_{2}$ & 0.973485 \\
$B_{1}$ & -0.062500 & $B_{5}$ & -0.043750 \\
$B_{2}$ & -0.115655 & $B_{6}$ & -0.018750 \\
$B_{3}$ & 0.064731 & $B_{7}$ & -0.479505 \\
$B_{4}$ & -0.072599 & $B_{8}$ & -0.233951 \\
\hline \hline
\end{tabular}

\section{THE HETEROCLINIC NETWORK}

The 4D system (1) with coefficients in Table II contains an attracting heteroclinic network whose structure is shown schematically in Fig. 3. We have verified this (a) by examination of the existence and stabilities of the equilibria from Table I, (b) by verification of robust connections, by examining eigenvalues, by direct (noise-free) simulation within invariant subspaces to ensure transverse intersections and by applying Theorem 1 from Ref. 20, and (c) by direct simulations using initial conditions away from invariant subspaces, both with and without added noise.

For direct simulation without noise the full network is not easily observable: apart from transients, the typical observed $\omega$-limit set is just the subcycle between $\xi_{\mathrm{LR}}$ and $\xi_{\mathrm{SS}}$. However, on addition of noise, one can observe much more of the network, including the excursions along the depth two connection. The LR/SS-subcycle is shown at the base of

TABLE III. Connections between equilibria listed in Table I. The third column indicates the equilibria contained within the unstable manifold of that equilibrium (i.e., for which there is a connecting trajectory), while the fourth column indicates the equilibria onto which the unstable manifold accumulates (i.e., lying in the closure of the unstable manifold).

\begin{tabular}{lccc}
\hline \hline Equilibrium & Dim of $W^{u}$ & $W^{u}$ connects to & $W^{u}$ accumulates on \\
\hline$\xi_{\mathrm{LR}}$ & 1 & $\xi_{\mathrm{SS}}$ & $\xi_{\mathrm{SS}}$ \\
$\xi_{\mathrm{SS}}$ & 2 & $\xi_{\mathrm{SR}}, \xi_{\mathrm{LR}}, \xi_{\mathrm{LS}}$ & $\xi_{\mathrm{SR}}, \xi_{\mathrm{LR}}, \xi_{\mathrm{LS}}$ \\
$\xi_{\mathrm{LS}}$ & 2 & $\xi_{\mathrm{LR}}, \xi_{\mathrm{SS}}, \xi_{\mathrm{WR}}$ & $\xi_{\mathrm{LR}}, \xi_{\mathrm{SS}}, \xi_{\mathrm{WR}}$ \\
$\xi_{\mathrm{SR}}$ & 2 & $\xi_{\mathrm{WR}}, \xi_{\mathrm{AR}}$ & $\xi_{\mathrm{WR}}, \xi_{\mathrm{AR}}, \xi_{\mathrm{LR}}, \xi_{\mathrm{SS}}$ \\
$\xi_{\mathrm{AR}}$ & 1 & $\ldots$ & $\xi_{\mathrm{WR}}, \xi_{\mathrm{LR}}, \xi_{\mathrm{SS}}$ \\
$\xi_{\mathrm{WR}}$ & 2 & $\ldots$ & $\xi_{\mathrm{LR}}, \xi_{\mathrm{SS}}$ \\
\hline \hline
\end{tabular}



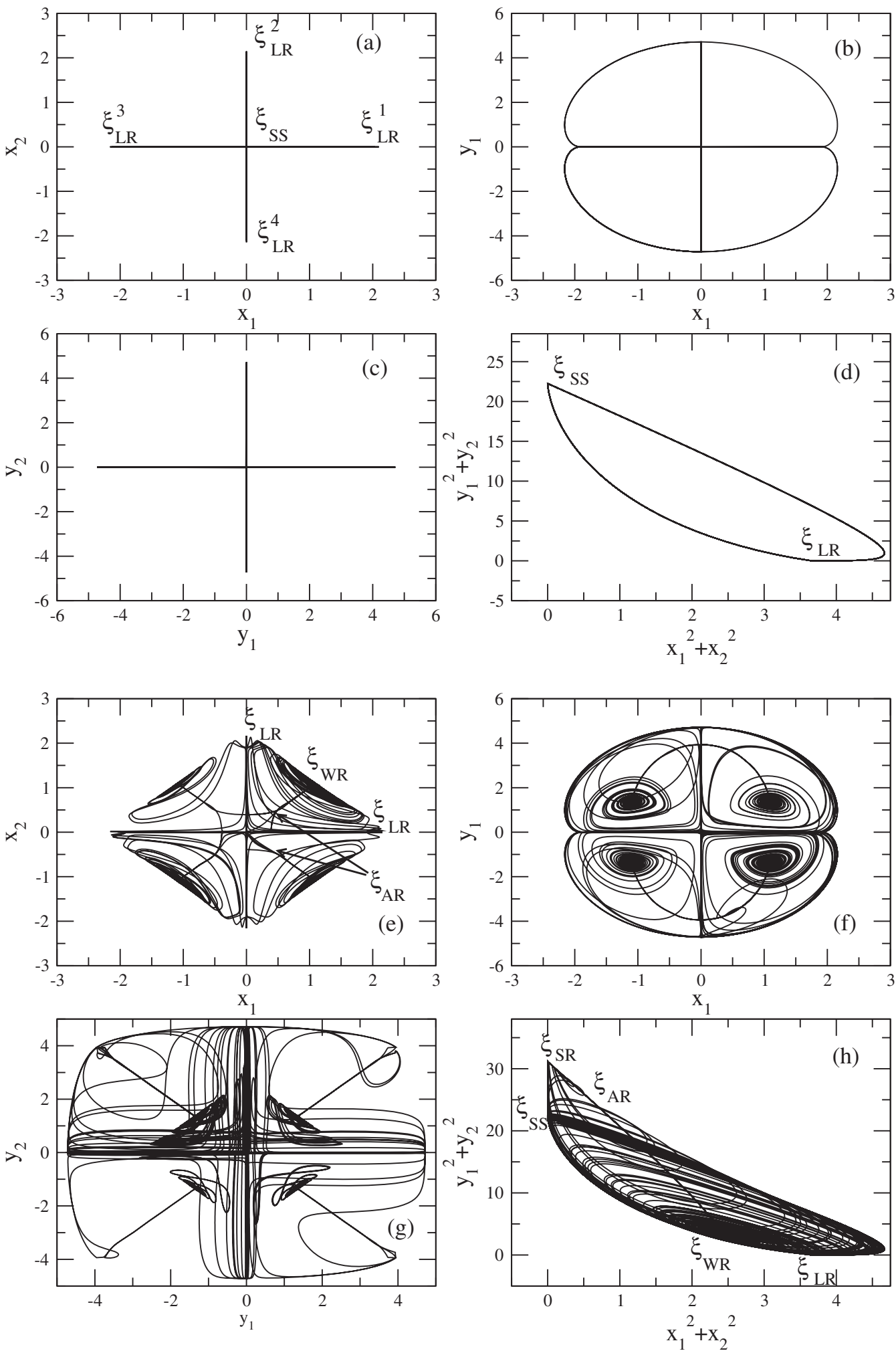

FIG. 2. Projections of a typical trajectory for the system (1) with parameters in Table II (a)-(d) for noise amplitudes $\epsilon_{1}=\epsilon_{2}=\epsilon_{3}=\epsilon_{4}=10^{-8}$ and (e)-(h) for $\epsilon_{1}=\epsilon_{2}=10^{-8}, \epsilon_{3}=\epsilon_{4}=10^{-3}$, after initial transients are allowed to decay. (a)-(d) show that the dynamics only explores symmetric copies of the subcycle between the four symmetric copies of $\xi_{\mathrm{LR}}$, while (e)-(h) show repeated excursions around a number of connections that accumulate back on the subcycle. Note that in projection [(a) and (e)] $\xi_{\mathrm{SR}}$ and $\xi_{\mathrm{SS}}$ are both projected onto the origin, and only a selection of equilibria is labeled. Panels (d) and (h) show the dynamics in a group invariant projection $\left(x_{1}^{2}+x_{2}^{2}, y_{1}^{2}+y_{2}^{2}\right)$ and distinguish all of the equilibria involved in the network. By contrast, projections of solutions where $\epsilon_{1}$ $=\epsilon_{2}=10^{-3}, \epsilon_{3}=\epsilon_{4} \leq 10^{-3}$ look very similar to (a)-(d) - the dynamics is much more sensitive to noise added to the last two components.

Fig. 3 while example trajectories are shown in phase space in Fig. 2 and time series in Fig. 4. Predominantly, an almost periodic temporal behavior is displayed in the latter figure that involves jumps between states where one of the $x_{i}$ is nonzero $\left(\xi_{\mathrm{LR}}\right.$ states) and states where one of the $y_{i}$ is nonzero ( $\xi_{\text {sS }}$ states). The approximate period decreases from (a) to (b) to (c) as the level of noise in the $y_{i}$ components is increased; the time series also show a transient. In addition (b) and (c) 


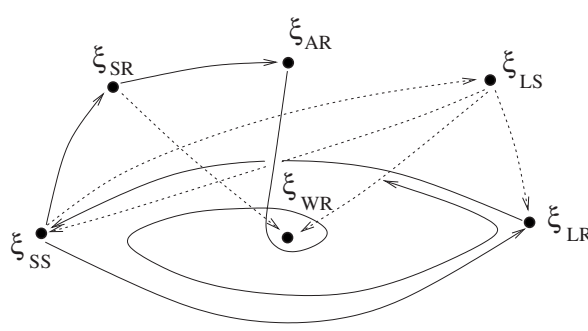

FIG. 3. Schematic diagram in a group invariant projection of phase space coordinates, showing the heteroclinic network for Eq. (1) with parameters as in Table II. This qualitative structure persists for an open set of nearby parameters. The dashed line connections are also present but are suppressed to emphasize the depth two connection back to the cycle. Note that the connection from $\xi_{A R}$ means the network is depth two, i.e., the unstable manifold of $\xi_{\mathrm{AR}}$ is not contained within the stable manifold of any cycle, but nevertheless it accumulates on the LR/SS subcycle between $\xi_{\mathrm{LR}}$ and $\xi_{\mathrm{SS}}$.

show excursions characterized by a distinctive pattern of long visits to an equilibrium where all $x$ 's and $y$ 's are nonzero, followed by a rapid and growing oscillation leading back to the LR/SS subcycle. These features of the attractors are visible in Fig. 2 where in (a)-(d) only the LR/SS subcycle is present, while in (e)-(h) the excursions also take place.

In the following Sec. II A, we perform an analysis of the dynamics near this attractor; we construct a local return map and show that the LR/SS subcycle is (a) a Milnor attractor and (b) the measure of its local basin of attraction includes all except a very small set in a neighborhood of the subcycle (i.e., it is essentially asymptotically stable)—recall that the local basin of attraction is the set of points whose trajectories remain near the cycle, and that are asymptotic to the cycle. We construct a return map for the excursions and use this to describe both deterministic and noise-induced excursions.

\section{A. Analysis of the heteroclinic attractor}

The LR/SS subcycle is a heteroclinic cycle

$$
\cdots \rightarrow \xi_{\mathrm{LR}} \rightarrow \xi_{\mathrm{SS}} \rightarrow \xi_{\mathrm{LR}} \rightarrow \cdots,
$$

where, in the coordinates $\left(x_{1}, x_{2}, y_{1}, y_{2}\right)$, the LR states are

$$
\xi_{\mathrm{LR}}^{1,2}=( \pm p, 0,0,0), \quad \xi_{\mathrm{LR}}^{3,4}=(0, \pm p, 0,0)
$$

and the SS states are

$$
\xi_{S S}^{1,2}=(0,0, \pm q, 0), \quad \xi_{S S}^{3,4}=(0,0,0, \pm q) .
$$

There is switching between the \pm images of these states in the presence of noise (see, for example, in Fig. 4(a), around 2500 time units) but we will identify all such points in the argument below.

We study the stability of this cycle by considering an approximate Poincaré map (similar to Refs. 1, 11, 13, 16, and 19) that is a composition of two local maps and two global maps between local sections as follows:

$$
\cdots H_{\mathrm{LR}}^{(\text {in) }} \stackrel{\phi_{1}}{\rightarrow} H_{\mathrm{LR}}^{\text {(out) }} \stackrel{\psi_{1}}{\rightarrow} H_{\mathrm{SS}}^{(\text {in) }} \stackrel{\phi_{2}}{\rightarrow} H_{\mathrm{SS}}^{(\text {out })} \stackrel{\psi_{2}}{\rightarrow} H_{\mathrm{LR}}^{(\text {in })} \cdots,
$$

where $H_{P Q}^{(\text {in) }}$ and $H_{P Q}^{(\text {out })}$ are local incoming/outgoing sections near $\xi_{P Q}$ (see Fig. 5). Near $\xi_{\mathrm{LR}}=(x, 0,0,0)$ we consider local coordinate $\left(u_{1}, z_{1}, v_{1}, w_{1}\right)$ aligned with the original coordi-
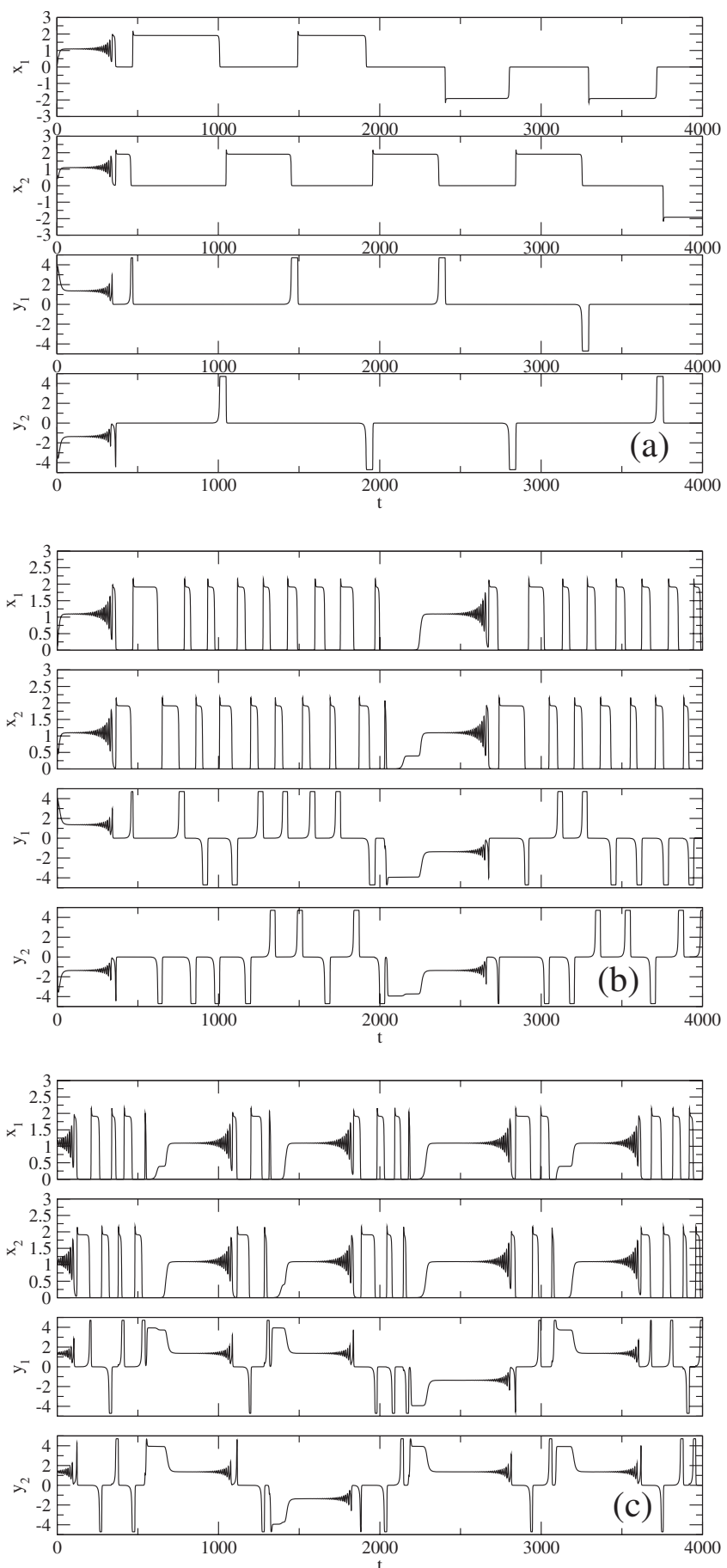

FIG. 4. Time series of $\left(x_{1}, x_{2}, y_{1}, y_{2}\right)$ showing trajectories in the presence of very low level anisotropic noise where (a) $\epsilon=\left(10^{-30}, 10^{-30}, 10^{-30}, 10^{-30}\right)$, (b) $\left(10^{-30}, 10^{-30}, 10^{-10}, 10^{-10}\right)$, and (c) $\left(10^{-30}, 10^{-30}, 10^{-5}, 10^{-5}\right)$. Observe the initial transient to the LR/SS subcycle in (a) (with random switching between which part of the unstable manifold is explored near the $\xi_{\mathrm{LR}}$ states), and in cases (b) and (c), increasingly anisotropic noise gives rise to more frequent excursions away from the LR/SS subcycle.

nate axes $\left(x_{1}, x_{2}, y_{1}, y_{2}\right)$. Assume that $\left\{\left|u_{1}\right|,\left|z_{1}\right|,\left|v_{1}\right|,\left|w_{1}\right| \leq 1\right\}$ is a scaling of a small neighborhood of $\xi_{\mathrm{LR}}^{1}$, where the behavior of the flow is almost linear, and define

$$
H_{\mathrm{LR}}^{(\mathrm{in})}=\left\{\left(u_{1}, z_{1}, v_{1}, w_{1}\right): u_{1}^{2}+v_{1}^{2}=1,\left|w_{1}\right|,\left|z_{1}\right| \leq 1\right\},
$$




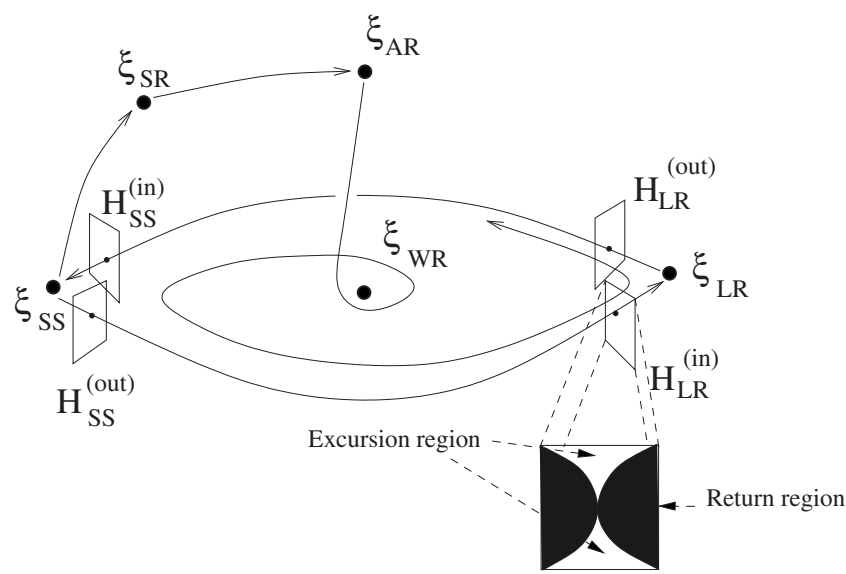

FIG. 5. Schematic diagram showing the sections used for calculating stability of the heteroclinic subcycle. The sections $H_{\mathrm{LR}, S \mathrm{~S}}^{\text {(in,out) }}$ are taken near representative equilibria $\xi_{\mathrm{LR}, \mathrm{SS}}$, and symmetrically related points are mapped to these points.

$$
H_{\mathrm{LR}}^{(\mathrm{out})}=\left\{\left(u_{1}, z_{1}, v_{1}, w_{1}\right):\left|u_{1}\right|,\left|v_{1}\right|,\left|z_{1}\right| \leq 1, w_{1}=1\right\} .
$$

The local map $\phi_{1}: H_{\mathrm{LR}}^{(\mathrm{in})} \rightarrow H_{\mathrm{LR}}^{(\mathrm{out})}$ to leading order can be expressed as

$$
\phi_{1}\left(u_{1}, z_{1}, v_{1}, w_{1}\right)=\left(u_{1} w_{1}^{r_{1} / e_{14}}, z_{1} w_{1}^{c_{12} / e_{14}}, v_{1} w_{1}^{c_{13} / e_{14}}, 1\right),
$$

where $r_{1}, e_{14}, c_{12}$, and $c_{13}$ are eigenvalues of linearization near $\xi_{\mathrm{LR}}$ and the respective values are given in Table I. We parametrize $H_{\mathrm{LR}}^{\text {(in) }}$ using $\left(\theta_{1}, w_{1}, z_{1}\right)$ by setting $u_{1}=\sin \left(\theta_{1}\right)$ and $v_{1}=\cos \left(\theta_{1}\right)$, and noting that generically we can assume that $\theta_{1} \notin(\pi / 2) Z$. For the mapping $\phi_{1}$ it is clear that, at leading order, only the coordinates $z$ and $w$ are significant for stability of the cycle. We identify symmetrically placed equilibria and illustrate these sections in Fig. 5.

Near $\xi_{\mathrm{SS}}=(0,0,0, y)$ we consider local coordinates $\left(v_{3}, w_{3}, z_{3}, u_{3}\right)$ aligned to the original coordinate axes $\left(x_{1}, x_{2}, y_{1}, y_{2}\right)$. The local map $\phi_{2}: H_{\mathrm{SS}}^{(\mathrm{in})} \rightarrow H_{\mathrm{SS}}^{(\mathrm{out})}$ is defined for

$$
H_{\mathrm{SS}}^{(\mathrm{in})}=\left\{\left(v_{3}, w_{3}, z_{3}, u_{3}\right): u_{3}^{2}+v_{3}^{2}=1,\left|w_{3}\right|,\left|z_{3}\right| \leq 1\right\}
$$

and

$$
H_{\mathrm{SS}}^{(\mathrm{out})}=\left\{\left(v_{3}, w_{3}, z_{3}, u_{3}\right):\left|u_{3}\right|,\left|v_{3}\right|,\left|z_{3}\right| \leq 1, w_{3}=1\right\},
$$

and to leading order this can be expressed as

$$
\phi_{2}\left(v_{3}, w_{3}, z_{3}, u_{3}\right)=\left(v_{3} w_{3}^{c_{41} / e_{24}}, 1, z_{3} w_{3}^{-e_{34} / e_{24}}, u_{3} w_{3}^{r_{4} / e_{24}}\right) .
$$

For $\phi_{2}$, as for $\phi_{1}$, only the coordinates $z$ and $w$ are significant for the stability of the cycle.

The leading order $(w, z)$ components of the global maps for the given $\xi_{\mathrm{LR}}, \xi_{\mathrm{SS}}$ are

$$
\psi_{1}: H_{\mathrm{LR}}^{(\mathrm{out})} \rightarrow H_{\mathrm{SS}}^{(\mathrm{in})} \quad \text { and } \quad \psi_{2}: H_{\mathrm{SS}}^{(\mathrm{out})} \rightarrow H_{\mathrm{LR}}^{(\mathrm{in})},
$$

and hence

$$
\begin{aligned}
& \left(w_{3}, z_{3}\right)=\psi_{1}^{(w, z)}\left(u_{1}, z_{1}, v_{1}, w_{1}\right)=\left(a_{1} z_{1}, b_{1} v_{1}\right), \\
& \left(w_{2}, z_{2}\right)=\psi_{2}^{(w, z)}\left(v_{3}, w_{3}, z_{3}, u_{3}\right)=\left(a_{4} z_{3}, b_{4} v_{3}\right),
\end{aligned}
$$

respectively, where $a_{j}$ and $b_{j}$ are constants. The $(w, z)$ components of the compositions $g_{1}=\psi_{1} \circ \phi_{1}: H_{\mathrm{LR}}^{(\mathrm{in})} \rightarrow H_{\mathrm{SS}}^{(\mathrm{in})}$ and $g_{2}$ $=\psi_{2}{ }^{\circ} \phi_{2}: H_{\mathrm{SS}}^{(\mathrm{in})} \rightarrow H_{\mathrm{LR}}^{(\mathrm{in})}$ to leading order are therefore

$$
\begin{aligned}
& \left(w_{2}, z_{2}\right)=g_{1}^{(w, z)}\left(w_{1}, z_{1}\right)=\left(\tilde{a}_{1} z_{1} w_{1}^{c_{12} / e_{14}}, \tilde{b}_{1} w_{1}^{c_{13} / e_{14}}\right), \\
& \left(w_{1}, z_{1}\right)=g_{2}^{(w, z)}\left(w_{2}, z_{2}\right)=\left(\tilde{a}_{4} z_{2} w_{2}^{-e_{34} / e_{24}}, \tilde{b}_{4} w_{2}^{c_{41} / e_{24}}\right),
\end{aligned}
$$

where $\tilde{a}_{j}$ and $\tilde{b}_{j}$ are bounded by constants. Defining logarithmic variables $\zeta_{j}=\ln z_{j}$ and $\eta_{j}=\ln w_{j}, j=1,2$, the mappings $g_{j}^{(w, z)}$ are, to leading order, linear,

$$
\left(\begin{array}{l}
\eta_{1}(t+1) \\
\zeta_{1}(t+1)
\end{array}\right)=M_{2}\left(\begin{array}{l}
\eta_{2} \\
\zeta_{2}
\end{array}\right)=M_{2} M_{1}\left(\begin{array}{l}
\eta_{1}(t) \\
\zeta_{1}(t)
\end{array}\right)
$$

with

$$
M_{1}=\left(\begin{array}{ll}
c_{12} / e_{14} & 1 \\
c_{13} / e_{14} & 0
\end{array}\right) \text { and } M_{2}=\left(\begin{array}{cc}
-c_{34} / e_{24} & 1 \\
c_{41} / e_{24} & 0
\end{array}\right) .
$$

The product $\quad M=M_{1} M_{2}: H_{\mathrm{SS}}^{(\mathrm{in})} \rightarrow H_{\mathrm{SS}}^{(\mathrm{in})} \quad($ or equivalently $\left.M_{2} M_{1}: H_{\mathrm{LR}}^{(\mathrm{in})} \rightarrow H_{\mathrm{LR}}^{(\mathrm{in})}\right)$ approximates the dynamics in logarithmic coordinates for a small neighborhood of the cycle. The matrices $M_{j}$ are sometimes called transition matrices, see, e.g., Ref. 16. To determine the local basin of attraction in a small neighborhood of a simple heteroclinic cycle [the subcycle (2) is simple], one needs to examine the eigenvalues $\lambda_{1}, \lambda_{2}$ and the associated eigenvectors $\left(v_{11}, v_{12}\right),\left(v_{21}, v_{22}\right)$ for the product $M$ of transition matrices. The eigenvalues of $M$ are 6.32 and 0.876 , with associated eigenvectors $(1,6.46)$ and $(1,0.384)$ [see Eq. (3) and Table I]. If both eigenvalues of a transition matrix are real, $\lambda_{1}>\lambda_{2}, \lambda_{1}>1, v_{11} v_{12}>0$, and $v_{21} v_{22}>0$, then it can be verified that

$$
\left\{(\zeta, \eta): \zeta \leq 0, \eta \leq 0,\left(v_{11} v_{22}-v_{11} v_{22}\right)^{-1}\left(v_{22} \zeta-v_{21} \eta\right)<0\right\}
$$

is the set of points in logarithmic coordinates whose trajectories are attracted to the cycle (more general results of this type are discussed in Ref. 19). Applying this to the matrix $M=M_{1} M_{2}$ we find that the starting points in $H_{\mathrm{SS}}^{\text {(in) }}$ for the trajectories attracted by the cycle satisfy $|w|<C_{1}|z|^{0.384}$. This means that along the connection $H_{\mathrm{LR}}^{(\text {out })} \rightarrow H_{\mathrm{SS}}^{(\text {in) }}$ the trajectories starting at the points satisfying $\left|x_{2}\right|<\widetilde{C}_{1}\left|y_{1}\right|^{0.384}$ are attracted by the cycle. Equivalently, points in $H_{\mathrm{LR}}^{(\mathrm{in})}$ that give rise to trajectories attracted by the cycle will satisfy to leading order $|w|>C_{2}|z|^{16.48}$. Hence, along the connection $H_{\mathrm{LR}}^{(\mathrm{out})} \rightarrow H_{\mathrm{LR}}^{(\mathrm{in})}$ the trajectories starting at the points satisfying

$$
\left|y_{2}\right|>\widetilde{C}_{2}\left|x_{2}\right|^{16.48}
$$

are attracted to the cycle. This implies that the LR/SS subcycle is essentially asymptotically stable, ${ }^{6}$ i.e., it attracts almost all trajectories starting in a sufficiently small neighborhood. Note that the high order of the power in Eq. (4) implies that the cusp of trajectories that escape from the LR/SS subcycle (see inset of Fig. 5) is extremely narrow.

\section{DYNAMICS IN THE PRESENCE OF ANISOTROPIC NOISE}

Note that isotropic noise is usually introduced as a modeling assumption; it is not invariant under rescaling, but is justified by the fact that for many systems the response depends only on the amplitude of the noise. More generally, it is justifiable to explore the dynamics of Eq. (1) under vary- 
TABLE IV. Frequency $F$ of excursions away from the LR/SS subcycle for Eq. (1) with parameters as in Table II. Note the monotonic increase of $F$ on increasing $\epsilon_{3,4}$, noise in the $y_{i}$ components. Note also the apparent cutoff below which the excursions become so infrequent that they are not apparent even for extended runs of $10^{5}$ time units or more. Note that excursions were not found in the isotropic case, even for quite high noise amplitudes.

\begin{tabular}{lcccc}
\hline \hline$\epsilon_{1}=\epsilon_{2}$ & $10^{-3}$ & $10^{-6}$ & $10^{-9}$ & $10^{-12}$ \\
$\epsilon_{3}=\epsilon_{4}$ & $10^{-3}$ & $10^{-6}$ & $10^{-9}$ & $10^{-12}$ \\
$F$ & 0 & 0 & 0 & 0 \\
$\epsilon_{1}=\epsilon_{2}$ & $10^{-12}$ & $10^{-12}$ & $10^{-12}$ & $10^{-12}$ \\
$\epsilon_{3}=\epsilon_{4}$ & $10^{-5}$ & $10^{-6}$ & $10^{-7}$ & $10^{-8}$ \\
$F$ & 0.030418 & 0.000311 & 0 & 0 \\
$\epsilon_{1}=\epsilon_{2}$ & 0 & 0 & 0 & 0 \\
$\epsilon_{3}=\epsilon_{4}$ & $10^{-4}$ & $10^{-8}$ & $10^{-10}$ & $10^{-12}$ \\
$F$ & 0.1456 & 0.03339 & 0.01859 & 0.0061 \\
$\epsilon_{1}=\epsilon_{2}$ & $10^{-30}$ & $10^{-30}$ & $10^{-30}$ & $10^{-30}$ \\
$\epsilon_{3}=\epsilon_{4}$ & $10^{-5}$ & $10^{-10}$ & $10^{-15}$ & $10^{-20}$ \\
$F$ & 0.14159 & 0.01869 & 0 & 0 \\
\hline \hline
\end{tabular}

ing assumptions on the vector $\left(\epsilon_{1}, \epsilon_{2}, \epsilon_{3}, \epsilon_{4}\right)$ of noise strengths, because the qualitative dynamics may crucially depend on details of these noise strengths.

\section{A. Numerical simulations}

The LR/SS subcycle (2) cannot be asymptotically stable because the unstable manifold of $\xi_{\mathrm{SS}}$ is two-dimensional and, in addition to the connection from $\xi_{S S}$ to $\xi_{\mathrm{LR}}$, there exists a connection from $\xi_{\mathrm{SS}}$ to $\xi_{\mathrm{SR}}$. For numerical simulations with isotropic added noise $\left(\epsilon_{1}=\epsilon_{2}=\epsilon_{3}=\epsilon_{4}\right)$, see Fig. 2(a); excursions are very rare after an initial transient - in fact they are not apparent even for very long simulations with isotropic noise of large amplitude.

Results of numerical simulations of the system (1) with coefficients given in Table II for varying levels of noise are shown in Fig. 4. Integration was performed using the RungeKutta integrator of XPPAUT (Ref. 9) with time step $h=0.05$ and additive Wiener noise, with some example trajectories checked using smaller time steps. Isotropic noise gives rise to trajectories that apparently remain on the LR/SS subcycle, while increasing noise in the $y_{i}$ components significantly increases the frequency of excursions away from the subcycle. Note also the changes in sign of the coordinates $x_{i}$ present in Fig. 4(a) and absent in (b) and (c); this happens when noise causes the trajectory to pass through the invariant subspaces $x_{i}=0$ in a neighborhood of the $\xi_{\mathrm{SS}}$ states where $x_{1}$ and $x_{2}$ are both close to zero.

Consider two sections to the flow, one $H_{\text {cyc }}$ that intersects the LR/SS subcycle once every cycle and another $H_{\text {exc }}$ that trajectories intersect once every excursion. Using this one can measure $F$, the frequency of excursions per cycle, that is, the average ratio of intersections with these two sections. Note that the quantity $F$ depends on the precise quantitative definition used for $H_{\text {cyc }}$ but the response of $F$ to varying noise levels should be qualitatively independent of precise definition used.

Table IV shows some numerically measured values for $F$ as a function of noise for the 4D model (1) with parameters as in Table II. The frequencies were calculated by taking a Poincaré section at $x_{1}^{2}+x_{2}^{2}=3$ and then noting that returns close to the LR/SS subcycle came back at $y_{1}^{2}+y_{2}^{2} \geq 9.83$, while the excursions correspond to cases where $y_{1}^{2}+y_{2}^{2}<9.83$. On taking a run of approximately 50000 time units the proportion $F$ of excursions per cycle were then calculated (discounting repeated intersections during one excursion). The average was calculated over approximately 500-1000 cycles, meaning that the credible accuracy of $F$ in Table IV is only three or four decimal places at most. Note that one can count excursions more simply by examining, for example, intersections with $y_{1}^{2}+y_{2}^{2}>23$ [see Fig. 2(h)]; however, this requires taking two sections and does not remove the problem that one can include an arbitrary number of oscillations as one moves away from $\xi_{\mathrm{WR}}$.

Note that the results in Table IV demonstrate the apparent lack of any excursions even for quite high amplitude of isotropic noise. A high level of anisotropy may be required to obtain excursions at all. Very long time series need to be computed as each cycle can take a long time, and in addition the excursions can occur very infrequently. Hence, we urge caution in interpreting the numerical values of $F$.

\section{B. Dynamics of the return map with noise}

Consider a small neighborhood of the equilibrium $\xi_{\mathrm{SS}}$ $=(0,0,0, y)$ in the local coordinates $\left(v_{3}, w_{3}, z_{3}, u_{3}\right)$. Without noise, the trajectories starting in the region

$$
\left\{(1-\delta)\left|w_{3}\right|^{e_{34} / e_{24}}>\left|z_{3}\right|\right\} \cap H_{\mathrm{SS}}^{(\mathrm{in})}
$$

leave the neighborhood in the direction of $\xi_{\mathrm{LR}}=(0, x, 0,0)$, and the ones starting in

$$
\left\{(1-\delta)\left|z_{3}\right|^{e_{24} / e_{34}}>\left|w_{3}\right|\right\} \cap H_{\mathrm{SS}}^{(\mathrm{in})}
$$

leave in the direction of $\xi_{\mathrm{SR}}=(0,0, y, y) .(\delta>0$ is a small constant, $e_{34}$ and $e_{24}$ are given in Table I, $e_{34} / e_{24} \approx 0.32$.)

Following Ref. 2, we assume that noise is significant only in neighborhoods of steady states (where the vector field is small) and that noise can be ignored outside these neighborhoods. The probability distribution of trajectories near a saddle equilibrium in a two-dimensional system was studied in the presence of noise in Ref. 24. It was shown that for an incoming normal distribution with a zero mean, the mean $\mu_{\text {exit }}$ and the standard deviation $\sigma_{\text {exit }}$ of the distribution of outgoing trajectories for asymptotically small noise will scale as

$$
\mu_{\text {exit }} \sim \epsilon^{\left|\lambda_{s}\right| / \lambda_{u}}, \quad \sigma_{\text {exit }} \sim \sqrt{c_{1} \epsilon^{2}+c_{2} \epsilon^{2\left|\lambda_{s}\right| / \lambda_{u}}},
$$

where $\lambda_{s}<0$ and $\lambda_{u}>0$ are the eigenvalues associated with the stable and the unstable directions of the saddle equilibrium, $\epsilon$ is the amplitude of the added noise, and $c_{1}, c_{2}$ are positive constants. For an equilibrium, where the saddle quantity $\lambda_{s}+\lambda_{u}$ is positive, $\mu_{\text {exit }}>\sigma_{\text {exit }}$ in the limit $\epsilon \rightarrow 0$, and hence outgoing trajectories are lifted away from the noisefree heteroclinic connection.

Consider a small neighborhood of the $\xi_{\mathrm{SS}}$; since noise is important only near equilibria, the probability distribution of trajectories approaching $H_{\mathrm{SS}}^{(\mathrm{in})}$ is essentially the same as in $H_{\mathrm{LR}}^{\text {(out) }}$ (up to a linear transformation). Similarly, consider a 

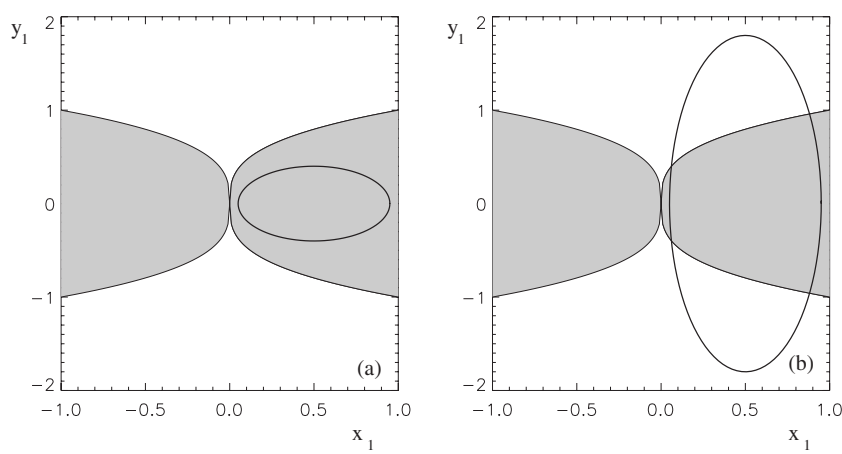

FIG. 6. Schematic diagram showing a "noise ellipse" indicative of the distribution of incoming trajectories in $H_{\mathrm{SS}}^{(\mathrm{in})}$ around the connection [here at $(0,0)]$ for the (a) isotropic and (b) anisotropic noise. The gray region shows the local basin of attraction of the LR/SS subcycle for the system in the absence of noise; the white region indicates where excursions via $\xi_{\mathrm{SR}}$ take place. The region of the noise ellipse that lies outside the gray region represents the probability of an excursion between two visits to the Poincaré surface of section.

small neighborhood of $\xi_{\mathrm{LR}}=(x, 0,0,0)$. In the subspace $\left(0, x_{2}, 0, y_{2}\right), \lambda_{s}=-c_{12} \approx-0.105, \lambda_{u}=e_{14} \approx 0.117$ (see Table I), and lift-off occurs. In the subspace $\left(0,0, y_{1}, y_{2}\right)$, $\lambda_{s}=-c_{13} \approx-0.782, \lambda_{u}=e_{14} \approx 0.117$, and so we expect no liftoff near the $\xi_{\mathrm{SS}}$ states.

Assume that the added noise is isotropic $\left(\epsilon_{1}=\epsilon_{2}=\epsilon_{3}=\epsilon_{4}\right)$ and so of equal amplitude in the $x_{i}$ and $y_{i}$ directions. Incoming trajectories in $H_{\mathrm{SS}}^{(\mathrm{in})}$ have a distribution with the mean $\left(\mu_{1}, \mu_{2}\right)$ and standard deviations $\left(\sigma_{1}, \sigma_{2}\right)$, schematically shown in Fig. 6 as an ellipse (drawn by a thick line) centered at $\left(\mu_{1}, \mu_{2}\right)$ with axes $\sigma_{1}$ and $\sigma_{2}$ (note that the ellipse represents the covariance ellipse of the noisy trajectories on return, not their support). The gray (white) area represents starting points for trajectories leaving the neighborhood in the direction of $\xi_{\mathrm{LR}}\left(\xi_{\mathrm{SS}}\right.$, respectively). Due to lift-off in the $\left(0, x_{2}, 0, y_{2}\right)$ subspace [schematically shown by the shift of the noise ellipse in the $x_{2}$ direction in Fig. 6(a)], added isotropic noise makes the LR/SS subcycle even more stable.

Now consider anisotropic noise $\left(\epsilon_{1}=\epsilon_{2} \ll \epsilon_{3}=\epsilon_{4}\right)$. On increase of $\epsilon_{3}$ relative to $\epsilon_{1}$, the vertical axis of the noise ellipse is stretched [see Fig. 6(b)], implying that anisotropic noise can make the LR/SS subcycle unstable. Note that the techniques of Ref. 24 assume an incoming normal distribution with a zero mean and suggest that this is a good model for small enough noise amplitudes; a more sophisticated model has been recently developed by Bakhtin. ${ }^{4}$

\section{A DEPTH TWO HETEROCLINIC NETWORK FOR CONVECTION}

Convection appears spontaneously when a horizontal layer of fluid, uniformly heated from below, commences motion by the uniform conducting state becoming unstable. The onset of convection is determined by the nondimensional Rayleigh number $R$ (which measures the relative amplitude of the buoyancy and the viscous forces) and the Prandtl number $P$ (which measures the ratio of viscous to thermal diffusion). In a previous paper ${ }^{20}$ we considered the nonlinear solutions near primary instability neighborhood of this mode interaction in $(R, k)$ space ( $R$ being the Rayleigh number and $k$ the horizontal wavenumber) for a variety of Prandtl numbers $P$.

In Ref. 20, we found parameter regimes where the only attractors are robust heteroclinic networks that can include connections from equilibria to subcycles by performing a reduction to a normal form that is an $8 \mathrm{D}$ system on $\mathrm{C}^{4}$. The latter system is detailed in the Appendix, Eq. (A1) with coefficients (A5), $\theta=1.34$ and Table VII; the Appendix shows that this system reduces to Eq. (1) on restricting to a $4 \mathrm{D}$ invariant subspace. As a consequence of Secs. II and III, we see that the essential dynamics is reproduced by the system reduced to an $\mathbb{R}^{4}$ invariant subspace, namely, Eq. (1).

We turn now to the full equations for Boussinesq thermal convection as governed by the Navier-Stokes equation

$$
\frac{\partial \mathbf{v}}{\partial t}=\mathbf{v} \times(\nabla \times \mathbf{v})+P \Delta \mathbf{v}+P R T \mathbf{e}_{3}-\nabla p,
$$

the incompressibility condition

$$
\nabla \cdot \mathbf{v}=0
$$

and the heat transfer equation

$$
\frac{\partial T}{\partial t}=-(\mathbf{v} \cdot \nabla) T+v_{3}+\Delta T,
$$

where $\mathbf{v}$ is the flow velocity and $T$ is the deviation of temperature of fluid from the linear profile in the trivial steady state. $R$ and $P$ are dimensionless parameters, the Rayleigh and Prandtl numbers, respectively. Stress-free horizontal boundaries held at fixed temperature are assumed,

$$
\frac{\partial v_{1}}{\partial x_{3}}=\frac{\partial v_{2}}{\partial x_{3}}=v_{3}=0, \quad T=0 \text { at } z=0,1 .
$$

The equations are solved numerically by applying standard pseudospectral methods. ${ }^{5}$ The fields are represented as a Fourier series,

$$
v_{j}=\sum_{\mathbf{n}} \hat{v}_{j, \mathbf{n}} \exp \left[\frac{2 \pi \mathrm{i}}{L}\left(n_{1} x_{1}+n_{2} x_{2}\right)\right] q^{j}\left(\pi n_{3} x_{3}\right),
$$

where $q^{1}\left(x_{3}\right)=q^{2}\left(x_{3}\right)=\cos x_{3}$ and $q^{3}\left(x_{3}\right)=q^{4}\left(x_{3}\right)=\sin x_{3}$ [here $\left.\mathbf{v}=\left(v_{1}, v_{2}, v_{3}\right), v_{4}=T\right]$. Substitution of Eq. (9) into Eqs. (5) and (7) yields a system of equations for the Fourier coefficients $w_{\mathbf{n}}^{j} \equiv \hat{v}_{\mathbf{n}}^{j}, j=1,2,3,4$ of the form

$$
\dot{w}_{j, \mathbf{n}}=F_{j, \mathbf{n}}(\mathbf{w}) \text {. }
$$

With an added random force the system of Eq. (10) takes the form

$$
\dot{w}_{j, \mathbf{n}}=F_{j, \mathbf{n}}(\mathbf{w})+\eta_{\mathbf{n}}^{j},
$$

where $\eta_{\mathbf{n}}^{j}$ are mutually independent white noise forcing components, $\left\langle\eta_{\mathbf{n}}^{i} \eta_{\mathbf{m}}^{j}\right\rangle=0$ for $i \neq j, \quad \mathbf{n} \neq \mathbf{m}$, and $\left\langle\eta_{\mathbf{n}}^{j}(t) \eta_{\mathbf{n}}^{j}\left(t^{\prime}\right)\right\rangle$ $=\left(\epsilon_{\mathbf{n}}^{j}\right)^{2} \delta\left(t-t^{\prime}\right)$. The forcing affects the first four spherical shells in the Fourier space $\left(\eta_{\mathbf{n}}^{j}=0\right.$ if $\left.n_{1}^{2}+n_{2}^{2}+n_{3}^{2}>4\right)$; its amplitude is

$$
\epsilon=\sum_{j, \mathbf{n}} \epsilon_{\mathbf{n}}^{j}
$$


Consider the symmetry $s$, defined by spatial translation by half a period with reference to both of the sides of the enclosing box. For symmetric fields, $v_{j, \mathbf{n}}=0$ with $n_{1}+n_{2}$ odd $(j=1, \ldots, 4)$; for antisymmetric fields, $v_{j, \mathbf{n}}=0$ with $n_{1}+n_{2}$ even $(j=1, \ldots, 4)$. Respectively, we introduce the amplitude of symmetric and antisymmetric parts of the added noise,

$$
\epsilon_{\mathrm{s}}=\sum_{j, \mathbf{n}, n_{1}+n_{2} \text { even }} \epsilon_{\mathbf{n}}^{j}, \quad \epsilon_{\mathrm{a}}=\sum_{j, \mathbf{n}, n_{1}+n_{2} \text { odd }} \epsilon_{\mathbf{n}}^{j} .
$$

The symmetry $s$ action on the center manifold coordinates is

$$
s:\left(z_{1}, z_{2}, z_{3}, z_{4}\right) \rightarrow\left(-z_{1},-z_{2}, z_{3}, z_{4}\right) .
$$

Hence, if random forcing $\eta$ in Eq. (11) is anisotropic with

$$
\frac{\epsilon_{s}}{\epsilon_{a}} \gg 1,
$$

its projections $P_{z_{j}}(\eta)$ along axes $z_{j}, j=1, \ldots, 4$, satisfy

$$
\left\langle\left(P_{z_{1}}(\eta)\right)^{2}\right\rangle=\left\langle\left(P_{z_{2}}(\eta)\right)^{2}\right\rangle \ll\left\langle\left(P_{z_{3}}(\eta)\right)^{2}\right\rangle=\left\langle\left(P_{z_{4}}(\eta)\right)^{2}\right\rangle,
$$

and in this sense we can make a heuristic identification $\epsilon_{a}$ $\sim \epsilon_{1}=\epsilon_{2}$ and $\epsilon_{s} \sim \epsilon_{3}=\epsilon_{4}$ in the $4 \mathrm{D}$ system (1). In the latter system, the bifurcation parameter is $\theta$ which can be related to the parameters of convection $R$ and $\delta=k-k_{m}$ as follows, where the other constants are given in the Appendix:

$$
\tan \theta=\frac{\lambda_{2}}{\lambda_{1}}=\frac{\beta_{21}+\beta_{22}\left(R-R_{m}\right) / \delta}{\beta_{11}+\beta_{12}\left(R-R_{m}\right) / \delta} .
$$

Note that the system we consider is constrained to have exact periodicity in both horizontal directions, and so will not be sensitive to long-wavelength modes that will be important for stability in an unconstrained setting.

We present numerical results for $\delta=-0.05 k_{m}$ and $R$ $=713$, corresponding to $\theta=1.34$. The convective attractor is the LR/SS subcycle, similar to that shown in Fig. 9a of Ref. 20. With an added stochastic forcing we observe excursions to $\xi_{\text {WR }}$ (e.g., as in Fig. 7), even when the amplitude of added noise is as small as $10^{-14}$. During the excursion a sample trajectory follows from the LR/SS subcycle to the LS/SS cycle, makes a revolution around it, then from $\xi_{\mathrm{SS}}$ it proceeds to $\xi_{\mathrm{SR}}$, followed by $\xi_{\mathrm{AR}}$ and afterward by $\xi_{\mathrm{WR}}$, from where it returns to the LR/SS cycle. It can be briefly described by the sequence of the steady states involved,

$$
\begin{aligned}
\cdots \xi_{\mathrm{LR}} & \rightarrow \xi_{\mathrm{SS}} \rightarrow \xi_{\mathrm{LS}} \rightarrow \xi_{\mathrm{SS}} \rightarrow \xi_{\mathrm{SR}} \\
& \rightarrow \xi_{\mathrm{AR}} \rightarrow \xi_{\mathrm{WR}} \rightarrow\left(\xi_{\mathrm{LR}} \rightarrow \xi_{\mathrm{SS}}\right) \cdots
\end{aligned}
$$

Other trajectories make two revolutions around the LR/SS cycle, or pass close to it. A different steady state $\xi_{\text {AS }}$ can be visited instead of $\xi_{\mathrm{AR}},{ }^{20}$ and a transition from $\xi_{\mathrm{SR}}$ to $\xi_{\mathrm{WR}}$ can occur, but the overall behavior is similar to that displayed in Fig. 7.

Table V shows numerically measured values for the frequency $F$ of excursions as a function of the noise amplitude for Boussinesq convection. Computations with anisotropic noise were done for two cases. If the added noise is isotropic, or when relatively large amplitude noise is added in the $\epsilon_{s}$ direction (anisotropic case I), generally speaking, the frequency of excursions decreases with the noise amplitude. By $\left|z_{1}\right|$
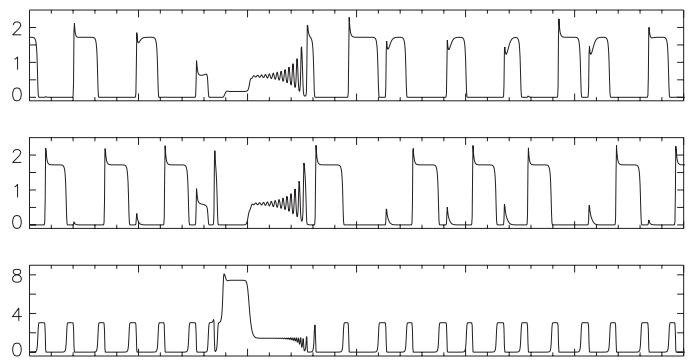

$\left|z_{4}\right|$
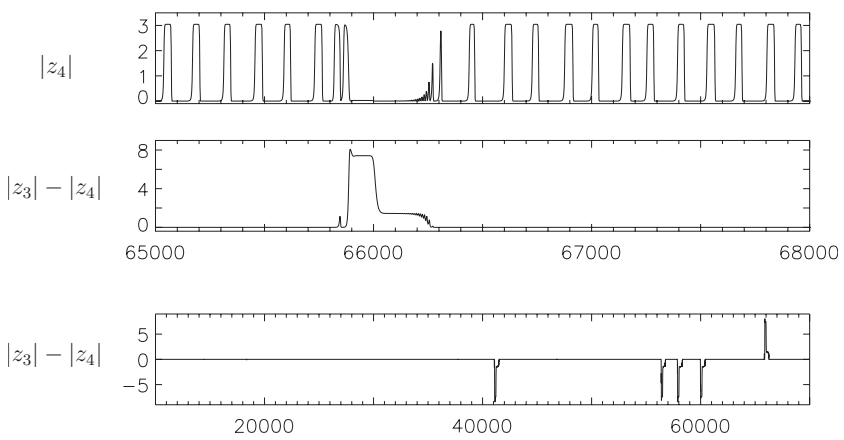

FIG. 7. Temporal evolution of $\left|z_{1}\right|,\left|z_{2}\right|,\left|z_{3}\right|,\left|z_{4}\right|$, and $\left|z_{3}\right|-\left|z_{4}\right|$ (the latter is shown twice on the last panel in a larger time interval) for convective attractors for $\delta=-0.05 k_{m}$ and $R=713$ and an added isotropic random forcing with amplitude $10^{-10} . z_{i}, i=1, \ldots, 4$ denote projections onto the basic vectors in the center eigenspace (see Appendix); the horizontal axis shows time.

contrast, if most of the noise is added in the $\epsilon_{a}$ direction (anisotropic case II), an opposite effect is observed as the amplitude of the added noise varies from $10^{-8}$ to $10^{-4}$; for smaller amplitudes, from $10^{-14}$ to $10^{-8}$, the variation with noise amplitude seems to be relatively weak. As discussed above, $\epsilon_{s}$ corresponds to addition of noise in the $z_{3}, z_{4}$ directions of the normal form at the mode interaction, and this corresponds to the $\epsilon_{3,4}$ amplitudes for the $4 \mathrm{D}$ model (1). As shown on the last panel of Fig. 7 (during an excursion the value $\left|z_{3}\right|-\left|z_{4}\right|$ is large, while otherwise it is close to zero), the distribution of excursions is nonuniform, and we presume that the time span of $2 \times 10^{5}$ of simulations is not sufficiently long to accumulate accurate statistics.

Although there are similarities as outlined above, there are clearly differences compared to the 4D system (see Sec. III); for example excursions in the 4D system are very rarely observed if the added noise is isotropic. This may be related to the fact that eigenvalues of linearizations (and the dimensions of corresponding eigenspaces) near the steady state $\xi_{\mathrm{LR}}$ in the two systems differ significantly. However, we have calculated the eigenvalues for the convective system on imposing the relevant symmetries, and find $e_{14}^{\text {conv }}=0.122, c_{12}^{\text {conv }}$ $=0.086$, and $c_{13}^{\text {conv }}=0.566$. The values are not very different to the ones given in Table I, and the arguments in Sec. III B about the lift-off within one of the invariant subspaces based on the differences of eigenvalues remain true. In addition, for convection the center manifold is properly $8 \mathrm{D}$ but we study in detailed dynamics only in a 4D subspace. We have performed simulations for the 8D normal form in the Appendix, and find the excursions are even rarer than for the 4D system. 
TABLE V. Frequency $F$ of excursions away from the LR/SS subcycle for the convection model for isotropic and for two anisotropic cases of added noise. Note that $F$ is small unless $\epsilon_{s}$ is relatively large.

\begin{tabular}{lcccccccc}
\hline \hline & $\epsilon=$ & $10^{-4}$ & $10^{-6}$ & $10^{-8}$ & $10^{-10}$ & $10^{-12}$ & $10^{-14}$ & $10^{-16}$ \\
\hline Isotropic & $\left(\epsilon_{s}=\epsilon_{a}=\epsilon\right)$ & 0.0103 & 0.0195 & 0.0100 & 0.0107 & 0.0036 & 0.0050 & 0 \\
Anisotropic I & $\left(\epsilon_{s}=\epsilon, \epsilon_{a}=10^{-12} \epsilon\right)$ & 0.0112 & 0.0139 & 0.0089 & 0.0065 & 0.0069 & 0.0043 & 0 \\
Anisotropic II & $\left(\epsilon_{s}=10^{-12} \epsilon, \epsilon_{a}=\epsilon\right)$ & 0.0007 & 0.0011 & 0.0057 & 0.0040 & 0.0108 & 0.0066 & 0 \\
\hline \hline
\end{tabular}

\section{DISCUSSION}

In the process of trying to understand the behavior of a depth two heteroclinic attractor we have found the first example with the minimum number of dimensions needed to see such behavior robustly in a symmetric system of ODEs. This behavior is not possible in three or fewer dimensions because, in order to connect robustly to a robust subcycle, it is necessary to have a three-dimensional proper invariant subspace for the dynamics.

Further study of the structure and dynamical properties of Eq. (1) at other parameter values is likely to bring other possible robust depth two network structures to light. In particular, for this example we find a subcycle that is essentially asymptotically stable, and hence excursions take place only as transients or in the presence of noise. This may no longer be the case if the coefficients $B_{i}$ are changed in a way that the subcycle ceases to be essentially asymptotically stablealthough in this case it is possible that this will destroy attraction for the network as a whole. Depth two cycles have been observed in other settings, ${ }^{3,8}$ but it is still unclear whether there are any robust examples such that typical trajectories in the noise-free case explore the whole network in their $\omega$-limit.

We observe that addition of noise can stabilize switching via "lift-off" of trajectories, as observed in Ref. 2. Depending on the details of the noise one may find either apparently deterministic cycling around the LR/SS subcycle or irregular excursions alternating with a fairly small numbers of cycles. This is dependent not just on the noise amplitude but also on the "shape" of the noise ellipse with respect to the local basin of attraction of the subcycle. Similar effects are presumably also observable for symmetry breaking perturbations of the network in the absence of noise ${ }^{12,18}$ including the possibility of chaotic dynamics near the network.

In terms of the Boussinesq convection model ${ }^{20}$ that originally motivated this study, we observe a depth two attractor with similar structure for numerical simulations in the presence of noise. The spatial-temporal structure of the noise is important in determining the frequency of excursions in that these depend on the noise strengths in the various spatial Fourier modes. We have not found a quantitative agreement of the frequency of excursions observed between the cycles from convection and that for the reduced model (1), but in fact there is no rigorous reason for the center manifold reduction to the truncated normal form (1) to be valid on addition of noise. For the 8D $\left(\mathrm{C}^{4}\right)$ model in Ref. 20 we find apparently far fewer excursions than in the example discussed here. This may be linked to the fact that $\xi_{\mathrm{SS}}$ has a three-dimensional unstable manifold for the $8 \mathrm{D}$ models, with a codimension two connection to $\xi_{\mathrm{SR}}$. By contrast, for the 4D model (1) the corresponding $\xi_{\mathrm{SS}}$ has an unstable manifold with a codimension one connection to $\xi_{\mathrm{SR}}$. It is an interesting open problem to understand the relative roles of noise amplitudes, eigenvalue, and dimensionality in determining the attraction properties and relative frequency of excursions for such robust attractors.

\section{ACKNOWLEDGMENTS}

We thank the Royal Society for partial support via a visitor grant for O.P. to visit Exeter in Spring 2008, during which time this work was commenced. The work of O.P. was partially supported by the Agence Nationale de la Recherche, France (Grant No. ANR-07-BLAN-0235 OTARIE) and by the Russian Foundation for Basic Research (Grant No. 0701-92217-CNRSL_a). We also thank Alastair Rucklidge for insightful comments on a draft of this paper.

\section{APPENDIX: REDUCTION FROM THE BOUSSINESQ NORMAL FORM}

As detailed in Ref. 20 the normal form on center manifold $\mathrm{C}^{4}$ with $\mathrm{D}_{4} \ltimes \mathbb{T}^{2} \times \mathrm{Z}_{2}$ symmetry truncated at cubic order can be written as

$$
\begin{aligned}
\dot{z}_{1}= & \lambda_{1} z_{1}+z_{1}\left(A_{1}\left|z_{1}\right|^{2}+A_{2}\left|z_{2}\right|^{2}+A_{3}\left(\left|z_{3}\right|^{2}+\left|z_{4}\right|^{2}\right)\right) \\
& +A_{4} \bar{z}_{1} z_{3} z_{4}, \\
\dot{z}_{2}= & \lambda_{1} z_{2}+z_{2}\left(A_{1}\left|z_{2}\right|^{2}+A_{2}\left|z_{1}\right|^{2}+A_{3}\left(\left|z_{3}\right|^{2}+\left|z_{4}\right|^{2}\right)\right) \\
& +A_{4} \bar{z}_{2} z_{3} \bar{z}_{4}, \\
\dot{z}_{3}= & \lambda_{2} z_{3}+z_{3}\left(A_{5}\left|z_{3}\right|^{2}+A_{6}\left|z_{4}\right|^{2}+A_{7}\left(\left|z_{1}\right|^{2}+\left|z_{2}\right|^{2}\right)\right) \\
& +A_{8}\left(z_{2}^{2} z_{4}+z_{1}^{2} \bar{z}_{4}\right), \\
\dot{z}_{4}= & \lambda_{2} z_{4}+z_{4}\left(A_{5}\left|z_{4}\right|^{2}+A_{6}\left|z_{3}\right|^{2}+A_{7}\left(\left|z_{1}\right|^{2}+\left|z_{2}\right|^{2}\right)\right) \\
& +A_{8}\left(\bar{z}_{2}^{2} z_{3}+z_{1}^{2} \bar{z}_{3}\right),
\end{aligned}
$$

where $A_{i}$ are real numbers (the normal form coefficients). The linear growth rates $\lambda_{1}$ and $\lambda_{2}$ are close to zero for the truncation to be valid; otherwise, higher order terms become important.

For the problem of Boussinesq convection in a planar region, these coefficients can be calculated as functions of the Rayleigh number $R$, the Prandtl number $P$, and the critical wavenumber $k$ by introducing 
TABLE VI. The coefficients $\alpha_{i j}$ and $\beta_{i j}$ in Eqs. (A3) and (A4) that determine the coefficients $A_{i}$ and $\lambda_{i}$ of the normal form Eq. (A1).

\begin{tabular}{lccc}
\hline \hline$\beta_{i j}$ & $j=1$ & $j=2$ & \\
\hline$i=1$ & 3.1551 & 0.01949 & \\
$i=2$ & -4.2921 & 0.02456 & $j=3$ \\
$\alpha_{i j}$ & $j=1$ & $j=2$ & -0.125 \\
\hline$i=1$ & 0 & 0 & -0.18081 \\
$i=2$ & -0.03207 & -0.01843 & -0.1744 \\
$i=3$ & 0.02281 & 0.13589 & -0.1441 \\
$i=4$ & -0.08110 & -0.3240 & -0.125 \\
$i=5$ & 0 & 0 & -0.17434 \\
$i=6$ & -0.03108 & -0.01958 & -0.2667 \\
$i=7$ & -0.1954 & -0.2513 & -0.1096 \\
$i=8$ & 0.1314 & 0.2237 & \\
\hline \hline
\end{tabular}

$$
\epsilon=R-R_{m}, \quad \delta=k-k_{m} .
$$

If we assume $\epsilon$ and $\delta$ are small then a center manifold approximation performed in the vicinity of $\left(k_{m}, R_{m}\right)$ is valid. Details of calculation of the normal form Eq. (A1) coefficients are given in Ref. 20 and are as

$\lambda_{i}=P(P+1)^{-1}\left(\beta_{i 1} \delta+\beta_{i 2} \epsilon\right)+O\left(\epsilon^{2}+\delta^{2}\right)$,

$A_{i}=P(P+1)^{-1}\left(P^{-2} \alpha_{i 1}+P^{-1} \alpha_{i 2}+\alpha_{i 3}\right)+O(|\epsilon|+|\delta|)$,

where the values of $\alpha_{i j}$ and $\beta_{i j}$ are listed in Table VI.

In addition to discrete symmetries, Eq. (A1) has a twodimensional group of continuous symmetries with their origins in spatial translations. These give some zero eigenvalues corresponding to group orbit directions and can be removed by a suitable change of coordinates; see, for instance, Ref. 20 for details.

We focus here on some particular dynamics of this normal form discovered in Ref. 20 by varying the two parameters $\left(\lambda_{1}, \lambda_{2}\right)$ that govern the growth rates as follows:

$$
\lambda_{1}=r \cos \theta, \quad \lambda_{2}=r \sin \theta .
$$

The parameter $\theta$ can be used to study the competition between the bifurcations to two different translation-invariant solutions (rolls parallel to the periodicity box or parallel to a diagonal) while the parameter $r>0$ gives the distance from the mode interaction. For $\theta \approx 1.34$ we find robust heteroclinic cycles with depth two connections. This paper relies on note that the main features of this robust heteroclinic attractor are present for the cycle restricted to the invariant subspace

$$
\left(z_{1}, z_{2}, z_{3}, z_{4}\right)=\left(x_{1}, x_{2}, i x_{3}, i x_{4}\right),
$$

and hence we examine the system (A6) below, in the main part of the paper, which on addition of noise is simply Eq. (A1) within this subspace,

$$
\begin{aligned}
& \dot{x}_{1}=\left(\lambda_{1}+A_{1} x_{1}^{2}+A_{2} x_{2}^{2}+A_{3}\left(x_{3}^{2}+x_{4}^{2}\right)-A_{4} x_{3} x_{4}\right) x_{1}, \\
& \dot{x}_{2}=\left(\lambda_{1}+A_{1} x_{2}^{2}+A_{2} x_{1}^{2}+A_{3}\left(x_{3}^{2}+x_{4}^{2}\right)+A_{4} x_{3} x_{4}\right) x_{2}, \\
& \dot{x}_{3}=\left(\lambda_{2}+A_{5} x_{3}^{2}+A_{6} x_{4}^{2}\right) x_{3}+\left(A_{7}\left(x_{1}^{2}+x_{2}^{2}\right)+A_{8}\left(x_{2}^{2}-x_{1}^{2}\right)\right) x_{4},
\end{aligned}
$$

TABLE VII. Parameter values for Eq. (A6) in the case of Prandtl number $P=1$, where we set $\left(\lambda_{1}, \lambda_{2}\right)=(r \cos \theta, r \sin \theta)$. The dynamics is clearly independent of $r>0$ (up to a time scaling), and hence the dynamics only depends on $\theta$. A detailed analysis of the bifurcations and attractors on varying $\theta$ and $P$ is detailed in Ref. 20.

\begin{tabular}{lllr}
\hline \hline$A_{1}$ & -0.062500 & $A_{2}$ & -0.115655 \\
$A_{3}$ & -0.007868 & $A_{4}$ & -0.274658 \\
$A_{5}$ & -0.062500 & $A_{6}$ & -0.112500 \\
$A_{7}$ & -0.356728 & $A_{8}$ & 0.122777 \\
\hline \hline
\end{tabular}

$$
\dot{x}_{4}=\left(\lambda_{2}+A_{5} x_{4}^{2}+A_{6} x_{3}^{2}\right) x_{4}+\left(A_{7}\left(x_{1}^{2}+x_{2}^{2}\right)+A_{8}\left(x_{2}^{2}-x_{1}^{2}\right)\right) x_{3} .
$$

Invariant subspaces and stabilities of relative equilibria within these subspaces are given in Table I in modified coordinates. In order to simplify further, we define a change of coordinates by

$$
\left(y_{1}, y_{2}\right)=\left(x_{3}+x_{4}, x_{3}-x_{4}\right),
$$

which places all of the subcycle equilibria on coordinate axes, where

$$
\begin{aligned}
& B_{1}=A_{1}, \quad B_{2}=A_{2}, \quad B_{3}=\frac{A_{3}}{2}-\frac{A_{4}}{4}, \\
& B_{4}=\frac{A_{3}}{2}+\frac{A_{4}}{4}, \quad B_{5}=\frac{A_{5}}{4}+\frac{A_{6}}{4}, \quad B_{6}=\frac{3 A_{5}}{4}-\frac{A_{6}}{4}, \\
& B_{7}=A_{7}-A_{8}, \quad B_{8}=A_{7}+A_{8} .
\end{aligned}
$$

It can be checked that Eq. (A6) can be rewritten as Eq. (1), and that the parameters in Table VII are transformed to those in Table II.

${ }^{1}$ Aguiar, M., Castro, S., and Labouriau, I., "Dynamics near a heteroclinic network," Nonlinearity 18, 391-414 (2005).

${ }^{2}$ Armbruster, D., Stone, E., and Kirk, V., "Noisy heteroclinic networks," Chaos 13, 71-79 (2003).

${ }^{3}$ Ashwin, P. and Field, M. J., "Heteroclinic networks in coupled cell systems," Arch. Ration. Mech. Anal. 148, 107-143 (1999).

${ }^{4}$ Bakhtin, Y., "Small noise limit for diffusions near heteroclinic networks," Dyn. Syst. (in press).

${ }^{5}$ Boyd, J. P., Chebyshev and Fourier Spectral Methods (Springer-Verlag, Berlin, 1989).

${ }^{6}$ Brannath, W., "Heteroclinic networks on the tetrahedron," Nonlinearity $\mathbf{7}$, 1367-1384 (1994).

'Chawanya, T., "Infinitely many attractors in a game dynamics system," Prog. Theor. Phys. 95, 679-684 (1996).

${ }^{8}$ Chawanya, T. and Ashwin, P., "A minimal system with a depth-two heteroclinic network, " Dyn. Syst. (in press).

${ }^{9}$ Ermentrout, G. B., XPPAUT (dynamical systems software). Available from http://www.math.pitt.edu/ bard/bardware/ (2000).

${ }^{10}$ Field, M. J., Lectures on Bifurcations, Dynamics and Symmetry, Pitman Research Notes in Mathematics Series Vol. 356 (Longmans, Green, New York, 1996).

${ }^{11}$ Hirschberg, P. and Knobloch, E., "A robust heteroclinic cycle in an $\mathbf{O}(2) \times \mathbf{Z}_{2}$ steady-state mode interaction," Nonlinearity 11, 89-104 (1998).

${ }^{12}$ Kirk, V. and Rucklidge, A. M., "The effect of symmetry breaking on the dynamics near a structurally stable heteroclinic cycle between equilibria and a periodic orbit," Dyn. Syst. 23, 43-74 (2008).

${ }^{13}$ Kirk, V. and Silber, M., "A competition between heteroclinic cycles," Nonlinearity 7, 1605-1621 (1994).

${ }^{14}$ Krupa, M., "Robust heteroclinic cycles," J. Nonlinear Sci. 7, 129-176 (1997).

${ }^{15}$ Krupa, M. and Melbourne, I., “Asymptotic stability of heteroclinic cycles 
in systems with symmetry," Ergod. Theory Dyn. Syst. 15, 121-148 (1995).

${ }^{16}$ Krupa, M. and Melbourne, I., "Asymptotic stability of heteroclinic cycles in systems with symmetry, II," Proc. - R. Soc. Edinburgh, Sect. A: Math. 134, 1177-1197 (2004).

${ }^{17}$ Krupa, M. and Melbourne, I., Nonasymptotic Stable Attractors in $O(2)$ Mode Interactions, Fields Institute Communications Vol. 4 (American Mathematical Society, Providence, 1995), pp. 219-232.

${ }^{18}$ Parker, M. J., Stewart, I. N., and Gomes, M. G. M., "Partial classification of heteroclinic behaviour associated with the perturbation of hexagonal planforms," Dyn. Syst. 23, 137-162 (2008).

${ }^{19}$ Podvigina, O. and Ashwin, P., "On local attraction properties and a stability index for heteroclinic connections," preprint, University of Exeter.

${ }^{20}$ Podvigina, O. and Ashwin, P., "The $1: \sqrt{2}$ mode interaction and heteroclinic networks in Boussinesq convection," Physica D 234, 23-48 (2007).
${ }^{21}$ Postlethwaite, C. M. and Dawes, J. H. P., "A codimension-two resonant bifurcation from a heteroclinic cycle with complex eigenvalues," Dyn. Syst. 21, 313-336 (2006).

${ }^{22}$ Proctor, M. R. E. and Matthews, P. C., " $\sqrt{2}: 1$ Resonance in nonBoussinesq convection," Physica D 97, 229-241 (1996).

${ }^{23}$ Recall that an invariant set $A$ is chain recurrent if given any $T>0, x, y$ in $A$, and $\epsilon>0$ there is a finite set of points $x=x_{0}, x_{1}, \ldots, x_{k}=y$ such that $\left|\Phi_{T}\left(x_{i}\right)-x_{i+1}\right|<\epsilon$ for $i=0,1, \ldots, k-1$.

${ }^{24}$ Stone, E. and Armbruster, D., "Noise and $\mathrm{O}(1)$ amplitude effects on heteroclinic cycles," Chaos 9, 499-506 (1999).

${ }^{25}$ Stone, E. and Holmes, P., "Random perturbations of heteroclinic attractors," SIAM J. Appl. Math. 50, 726-743 (1990).

${ }^{26}$ The original normal form coordinates in Ref. 20 are related to ours by $\left(x_{1}, x_{2}, i\left(y_{1}+y_{2}\right) / 2, i\left(y_{1}-y_{2}\right) / 2\right)$. A brief summary of the motivation and the steps involved in the reduction are given below and in Sec. IV with further details in the Appendix. 